\section{SAICAR drives cancer cell}

\section{survival}

Glucose metabolism is often altered in cancer to promote cell survival under stress. SAICAR (succinylaminoimidazolecarboxamide ribose5 '-phosphate) is an intermediate of de novo purine nucleotide synthesis. Lee and colleagues report that this metabolite stimulates pyruvate kinase isoform M2 (PKM2), a glycolytic enzyme crucial in the metabolic reprogramming of cancer cells towards growth and survival when glucose is scarce (Science 338, 1069-1072; 2012).

The authors tested metabolites from lung cancer cells for their ability to interact with PKM2 and identified SAICAR as a compound binding to PKM2 under low glucose conditions. Synthetic SAICAR was shown to stimulate the pyruvate kinase activity of PKM2, and this metabolite was elevated and able to activate PKM2 in glucose-deprived human cancer cell lines. Depletion of PAICS and ADSL, the SAICAR synthesizing and cleaving enzymes, respectively, showed that SAICAR levels affected glucose metabolism as well as cancer cell proliferation and survival in glucoselimiting situations. To demonstrate the functional relevance of the SAICAR-PKM2 interaction, the authors designed a PKM2 point mutant that was refractory to the effects of SAICAR. They showed that, in contrast to wild-type PKM2, expression of this mutant was not able to sustain the survival of cancer cells proficient in SAICAR production but depleted of endogenous PKM2, demonstrating that the SAICAR-PKM2 interaction was necessary for cancer cell survival in glucose-depleted settings.

These findings reveal an additional layer in the regulation of the metabolic reprogramming of cancer cells.

AIZ

\section{Mad1 doubles as nuclear import regulator}

In budding yeast cells, where mitosis occurs with an intact nuclear envelope, structural changes in the nuclear pore complex (NPC) during mitosis cause inhibition of Kap121mediated nuclear import. Wozniak and colleagues now identify the checkpoint protein Mad1 as a critical mediator of this inhibition (Mol. Cell http://doi.org/jx2; 2012).

The authors first established that import of a Kap121 reporter is lost when microtubules are destabilized, or when microtubule-kinetochore attachments are disrupted. Mad1 and Mad2 are components of the spindle assembly checkpoint (SAC) that senses unattached kinetochores, and are known to bind NPCs. They found that Mad1, but not Mad2, is needed for shutting off import in mitosis, and that this requires the binding of Mad1 to both kinetochores and NPCs, and the exchange of Mad1 between these pools. The kinase Ipl1 (also known as Aurora B) is a regulator of kinetochore-microtubule attachments, and the authors determined that Ipl1 is also required for

\title{
The haematopoiesis transcription landscape
}

Transcriptional profiling of adult haematopoietic stem cells (HSCs) and their differentiating daughters has given insights into lineage fate choice. So far, analysis of the expression program of haematopoetic stem cells arising during development has been lacking, partly because sufficient knowledge has only recently been gathered on the ontogeny of haematopoietic stem cells to allow their isolation from embryos.

Daley and colleagues (Cell Stem Cell 11, 710-714; 2012) have used surface markers to isolate emerging HSCs from the haematopoietic sites of developing embryos, including the yolk sac, placenta and ventral dorsal aorta, as well as from adult bone marrow and embryonic stem cells differentiating to embryoid bodies. The authors combined genome-wide expression profiling with gene regulatory network analysis to delineate three distinct global transcriptional states during haematopoietic development. They observed that HSCs emerging from the dorsal aorta initially exhibit a gene expression profile close to that of macrophages. They also uncovered that HSCs derived from embryoid bodies, despite displaying a transcription program most related to definitive HSCs from the bone marrow, seem deficient in Notch signalling, perhaps explaining their aberrant lymphopoiesis in functional tests. Finally, the authors use their data to identify known and novel transcriptional regulators of HSC ontogeny, which they functionally test using a loss-of-function approach in zebrafish. This resource will be useful to understand developmental haematopoiesis and to plan direct differentiation of pluripotent cells to HSCs for eventual therapeutic purposes.

the inhibition of the Kap121 import pathway. They go on to show that the nuclear level of the protein phosphatase Glc7, an antagonist of Ipl1, is regulated by Kap121 and Mad1, and suggest that inhibition of this transport pathway may support the function of Ipl1 during SAC arrest.

It remains to be investigated whether inhibition of nuclear import occurs in other organisms in early stages of mitosis, before the nuclear envelope is disassembled. CKR

\section{Inducing necrosis with SIRTainty}

Tumor necrosis factor- $\alpha$ (TNF- $\alpha$ ) elicits programmed necrosis, or necroptosis, by stimulating an interaction between the RIP1 and RIP3 kinases. However, the mechanisms regulating RIP1-RIP3 complex formation and the induction of necroposis remain incompletely defined. Finkel and colleagues report that the deacetylase SIRT2 has an important role in these processes (Nature http://doi.org/jx3; 2012).

The authors found that SIRT2 interacts directly with RIP3 in vitro and in vivo. Intriguingly, SIRT2 deletion or depletion blocked TNF-a-stimulated RIP1-RIP3 complex formation and necroptosis induction. Similar results were observed on treatment with the SIRT2 deacetylase inhibitor AGK2, suggesting that the catalytic activity of SIRT2 is critical for these effects.

Indeed, RIP1 is acetylated under basal conditions, but acetylation decreased following TNF- $\alpha$ treatment. RIP1 was shown to be a direct target of SIRT2, and knocking down SIRT2 or expressing a catalytically inactive SIRT2 mutant attenuated RIP1 deacetylation in vivo. Moreover, an acetylation-deficient RIP1 mutant formed a constitutive complex with RIP3 and efficiently induced necroptosis following TNF- $\alpha$ treatment.

Finkel and colleagues also showed that Sirt2 loss or inhibition promoted recovery in a mouse model of ischemia-reperfusion injury, where blocking necrosis is known to have a protective effect. Their findings provide fresh insight into the mechanisms regulating necroptosis, and suggest that SIRT2 inhibition might be a clinically relevant strategy to treat diseases involving necrosis. EJC

By Nathalie Le Bot, Emily J. Chenette, Christina Karlsson Rosenthal and Alexia-Ileana Zaromytidou 